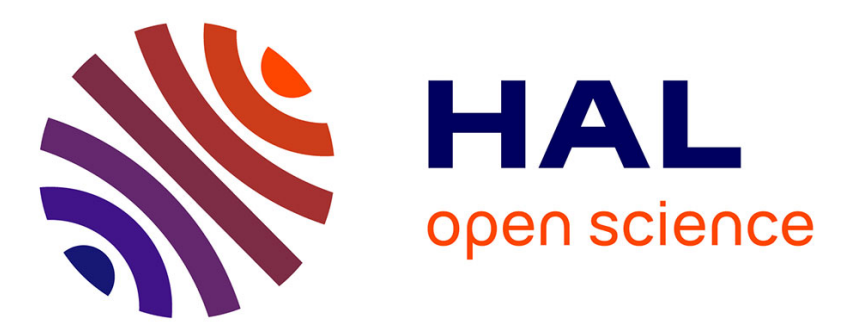

\title{
Experimental investigation of the effect of thresholding on temporal statistics of avalanches
}

\author{
M A Lebyodkin, I V Shashkov, T A Lebedkina, V S Gornakov
}

\section{To cite this version:}

M A Lebyodkin, I V Shashkov, T A Lebedkina, V S Gornakov. Experimental investigation of the effect of thresholding on temporal statistics of avalanches. Physical Review E , 2017, 95, pp.032910. 10.1103/physreve.95.032910 . hal-03256678

\section{HAL Id: hal-03256678 \\ https://hal.univ-lorraine.fr/hal-03256678}

Submitted on 15 Nov 2021

HAL is a multi-disciplinary open access archive for the deposit and dissemination of scientific research documents, whether they are published or not. The documents may come from teaching and research institutions in France or abroad, or from public or private research centers.
L'archive ouverte pluridisciplinaire HAL, est destinée au dépôt et à la diffusion de documents scientifiques de niveau recherche, publiés ou non, émanant des établissements d'enseignement et de recherche français ou étrangers, des laboratoires publics ou privés. 
PHYSICAL REVIEW E 95, 032910 (2017)

DOI: 10.1103/PhysRevE.95.032910

\title{
Experimental investigation of the effect of thresholding on temporal statistics of avalanches
}

\author{
M.A. Lebyodkin, ${ }^{1 *}$ I.V. Shashkov, ${ }^{2}$ T.A. Lebedkina, ${ }^{1}$ V.S. Gornakov ${ }^{2}$ \\ ${ }^{1}$ Laboratoire d'Etude des Microstructures et de Mécanique des Matériaux, UMR 7239, \\ CNRS/Université de Lorraine, Ile du Saulcy, 57045 Metz, France \\ ${ }^{2}$ Institute of Solid State Physics RAS, 142432 Chernogolovka, Russia
}

\begin{abstract}
Avalanche-like behavior reflected in power-law statistics is a ubiquitous property of extended systems addressed in a number of generic models. The paper presents an experimental investigation of the effect of thresholding on the statistics of durations and waiting times between avalanches using acoustic emission accompanying unstable plastic deformation. It is found that durations of acoustic events obey power-law statistical distributions robust against thresholding. The quiescent time distributions follow the Poisson law for low threshold values. Both these results corroborate the hypothesis that plastic deformation is akin to the phenomena associated with self-organized criticality (SOC), often advanced on the basis of power-law amplitude statistics. Increasing the threshold height enforces deviation from the Poisson distributions toward apparent power-law behavior. Such a thresholding effect may hinder the experimental determination of SOC-like dynamics because of the inevitable noise.
\end{abstract}

PACS numbers: 45.70.Ht, 62.20.F-, 05.65.+b, 43.60.+d

* Corresponding author. E-mail address: mikhail.lebedkin@univ-lorraine.fr

\section{INTRODUCTION}

Many nonlinear dynamical systems display an intermittent response to smooth driving. Numerous examples include the Barkhausen noise in magnetic materials [1], vortex avalanches in superconductors [2], earthquakes [3], dry friction [4], crack growth [5], deformation of both crystalline [6] and non-crystalline solids [7,8], and others (see also reviews [9-12]). All these phenomena are characterized by avalanche-like relaxation processes complying with scale-free 
power-law distributions of the avalanche sizes (energies) and durations. To explain the abundance of such behaviors in natural systems, the concept of self-organized criticality (SOC) is often appealed to $[9,13]$. It claims that a complex system slowly driven by an uncorrelated signal can selforganize to a critical state without fine tuning of the order parameter. The assumption of uncorrelated loading at a vanishing rate implies independent avalanche nucleation and allows for avoiding superposition of avalanches. Therefore, a Poisson-like exponential law can be expected for the statistics of waiting times between consecutive avalanches. However, power-law distributions were found in many experiments, thus questioning the SOC mechanism, e.g., for the turbulent transport in the magnetically confined plasma, solar fluxes, earthquakes, or paper fracture experiments [14-17]. At the same time, numerous theoretical works showed that power-law statistics of quiescent times can appear in systems governed by the SOC dynamics. In particular, it can happen when the statistics of the avalanche returns is gathered locally $[18,19]$, in the case of a correlated driving signal [20], or because of temporal variations of the activity rate [21]. Importantly, power-law distributions can also occur when only avalanches with size above some threshold are analyzed [22-24]. The last property suggests a general mechanism governing powerlaw behavior of experimental data which are inevitably corrupted by noise and require thresholding to extract individual events [25]. In particular, numerical analysis of the effect of noise addition to models characterized by avalanche dynamics leads to prediction of a transition from the Poisson to power-law statistics [24]. The first experimental evidence for this prediction was found very recently by visualization of crack propagation [26].

This work reports on the first experimental investigation of the thresholding effect using acoustic emission (AE) accompanying plastic deformation of crystals. Governed by the motion of dislocations and their interaction with each other and with other crystal defects, plastic deformation is an inherently collective process often associated with power-law statistics. Remarkably, the collective nature of the dislocation dynamics sometimes leads to macroscopically unstable plastic flow giving rise to serrations of the applied force. Scale-free statistics of the force serrations were indeed detected under certain conditions for various mechanisms of plastic instability [6,27-29]. Even though plastic flow of most materials is macroscopically stable, so that smooth force-time curves are usually observed, ubiquitous power-law behaviors were found for both stable and unstable flow in a large range of mesoscopic scales accessible to experimental techniques based on the recording of electric [30,31] or, more recently, AE signals [32-37]. In the present work, AE is analyzed under conditions of plastic instability governed by the Portevin-Le Chatelier (PLC) effect [38]. This choice is justified by a high activity of AE during the PLC effect [36,37], which allows for varying the threshold in a relatively large range, while keeping enough events in each statistical sample. Moreover, it was found recently that the threshold height has a weak effect on the powerlaw indices of the corresponding $\mathrm{AE}$ amplitude distributions [39]. It should be noted that most of the literature data on the $\mathrm{AE}$ statistics during plastic deformation concerned the $\mathrm{AE}$ events amplitudes (or energy). The analysis of temporal parameters remained marginal because they are more strongly affected by diverse factors, such as the avalanche overlapping, sound reflections, occurrence of aftershocks, or background noise. For this reason, the present work is devoted to the study of both durations and waiting times of AE events, in order to verify the hypothesis of SOC for the dislocation dynamics.

\section{EXPERIMENTAL TECHNIQUE AND DATA PROCESSING}

Polycrystalline Al-5 wt $\% \mathrm{Mg}$ specimens with a gauge part $30 \times 7 \times 1 \mathrm{~mm}^{3}$ and an average grain size about 4-6 $\mu \mathrm{m}$ were deformed by tension at room temperature and constant grip velocity corresponding to the initial applied strain rate $\dot{\varepsilon}_{a}$ varied in the range of $2 \times 10^{-5} \mathrm{~s}^{-1}$ to $6 \times 10^{-3} \mathrm{~s}^{-1}$. Details of mechanical tests were reported elsewhere [36]. The specimens were deformed until fracture which is accompanied with an outstanding $\mathrm{AE}$ event used to synchronize the deformation curve with the AE signal. The AE was captured by a piezoelectric transducer clamped to the specimen surface just above the gauge part, so that it gathered the "global" AE signal as required in 
SOC models. In contrast to the usual experimental scheme of real-time extraction of AE events using preset criteria, the signal was recorded continuously at a sampling rate of $2 \mathrm{MHz}$. Each record could then be processed to generate various series of acoustic events ("hits") by varying the criteria which were chosen to be the same as in the standard real-time procedure [36,39]. Namely, the starting time $t_{s}$ fixes the instant when the absolute value of the signal surpasses the threshold voltage $V_{0}$. The ending time $t_{e}$ corresponds to the instant after which the signal remains below $V_{0}$ longer than for the hit definition time (HDT). Then, no measurements are performed during a hit lockout time (HLT), or "dead time," in order to filter out sound reflections. It is obvious that HLT may result in a loss of a part of the useful signal, particularly, "aftershocks." However, it was found earlier that the energy statistics of $\mathrm{AE}$ is rather robust against variation of various parameters including HLT [39]. For the purpose of this paper, all above-introduced parameters were chosen in the ranges corresponding to robust power-law energy distributions. The values HDT $=30 \mu$ s and HLT $=100 \mu \mathrm{s}$ were taken as the basic set. Another set with overstated values, HDT $=$ HLT $=300$ $\mu \mathrm{s}$, was used in control calculations.

The effect of $V_{0}$ variation on the determination of avalanches for the same choice of HDT and HLT is clarified in Fig. 1. For the sake of illustration, this scheme shows a portion of the signal where the $\mathrm{AE}$ is virtually continuous and the events only slightly exceed the noise background, in order to make visible the carrier oscillations. The real signal-to-noise ratio in the analyzed time spans exceeded at least two orders of magnitude (see, e.g., Fig. 2). The threshold of approximately $1.2 \mathrm{mV}$ was the lowest level below which individual events could not be extracted from the continuously recorded data stream because of the false connection by noise. Accordingly, the minimum threshold of $1.22 \mathrm{mV}$ was used. This value corresponds to the noise level of $25 \mathrm{~dB}$ obtained in blank measurements and allowed for an additional check by comparing the statistical results with a similar analysis of events extracted in real time by the device operating on a logarithmic scale. Such verification revealed a very good identity of the calculation results.

The duration $\delta$ of the $n$th event is given by $\delta(n)=t_{e}(n)-t_{s}(n)$. As far as the waiting time $t_{w}(n$, $n+1)$ is concerned, the following three definitions were suggested in the literature: $t_{w}=t_{s}(n+1)$ $t_{s}(n), t_{w}=t_{p}(n+1)-t_{p}(n)$, and $t_{w}=t_{s}(n+1)-t_{e}(n)$, where $t_{p}$ corresponds to the event peak amplitude. Following the arguments [20], we adopted the last definition corresponding to the interval between the end of one hit and the beginning of the next one. This approach avoids mixing $t_{w}$ with $\delta$, taking into account that $\delta$ is distributed according to a power law in the case of SOC and, therefore, may bias the results of the analysis of $t_{w}$. It should be noted that the HLT determines the low limit of detection of the waiting time.

For the sake of an intuitively clear demonstration of the thresholding effect, the results of calculation of discrete frequency distributions of $\delta$ and $t_{w}$ will be presented in the next section. Previous studies [36,37,39] showed that with some precautions described below, this traditional method provides a reasonable evaluation of the power-law exponents for AE accompanying the PLC effect, consistent with those obtained by rigorous approaches such as maximum-likelihood estimation (MLE) methods with goodness-of-fit tests suggested recently [40,41]. For comparison, power-law exponents obtained by these methods will also be given. The frequency distributions were calculated using data rescaled by the average value of the studied quantity. This approach was applied in order to avoid arbitrariness in the choice of the bin size and utilize a unique bin for all calculations. The least statistical samples corresponding to the highest threshold comprised more than 700 data values. Similar to our previous works, linear binning was used [36,37]. Accordingly, to handle the statistics of rare large-scale events, the initial-size bins containing fewer events than a preset minimum were merged with the right-hand neighbors until this minimum number (five in the present work) was reached. The obtained numbers were then normalized by the resulting bin sizes to calculate the probability density functions (PDF).

\section{EXPERIMENTAL RESULTS AND DISCUSSION}

Figure 2 presents examples of a portion of a force-time deformation curve and the accompanying AE signal. Among other things, it illustrates a general difficulty associated with the investigations 
of the collective dislocation dynamics. Namely, plastic flow is not a stationary process but implies strain hardening that is caused by the generation and storage of dislocations and the resulting evolution of the microstructure. Indeed, it can be seen that the applied force, the force serrations, and the acoustic signal evolve during deformation. Moreover, it has been shown that the AE statistics also evolve in the case of the PLC effect [36]. Namely, although the AE energy distributions manifest ubiquitous power-law behavior, the exponents found for short enough intervals where AE may be considered as quasi-stationary are generally not unique. For this reason, the strategy proposed in Refs. [36,39] was adapted for the present analysis. More specifically, the entire time interval was subdivided into short enough periods within which the power-law indices of energy and duration distributions remained unchanged upon further subdivision. The arrows traced in Fig. 2 indicate the time interval used to obtain the examples of analysis presented in Figs. 3 and 4(c-d). Although relatively low at this stage of deformation, the maximum amplitudes of AE events reach $170 \mathrm{mV}$ in this interval and exceed the maximum noise level by more than two orders of magnitude. It should be underlined that in spite of not obligatorily the same power-law exponents, the thresholding effect was similar for datasets selected on various deformation stages. This robustness justifies the approach adapted in view of the objective of the present work, that the calculations of the statistical distributions were performed using raw signals in order to avoid any effect of the signal pretreatment, even if denoising might improve the detection of authentic power laws (see, e.g. [42]).

Figure 3 represents examples of the effect of $V_{0}$ on the distributions of event durations for $\dot{\varepsilon}_{a}=2 \times 10^{-4} \mathrm{~s}^{-1}$ and two choices of time settings. Similar behavior of event duration distributions was found in all experimental conditions, even for the highest strain rate characterized by strong overlapping of AE events, which could have biased the analysis results [39]. It can be seen in the case of the relatively small HDT (left plot) that the PDF follows an accurate power-law dependence, $P(\delta /<\delta>) \sim(\delta /<\delta>)^{-\tau}$, over more than two orders of magnitude of the normalized duration. The dependences obtained for various $V_{0}$ agree quite well with each other although the size of the statistical sample is reduced from $N=1.4 \times 10^{5}$ to $10^{4}$ events, respectively, when $V_{0}$ is increased from the least value to $3.05 \mathrm{mV}$. As discussed in [40], such robustness with regard to the number of events is characteristic of power-law statistics. The estimates of the slope $\tau$ are synthesized in Table I. The data obtained for intermediate threshold heights testify that $\tau$ lies approximately between 2.2 and 2.3. It decreases because of either merging of individual avalanches, when $V_{0}$ is too low, or removal of small avalanches at high $V_{0}$, both factors causing higher probability of longer events. Table I also shows that the MLE method renders similar estimates. The comparison of data in the second and third columns confirms that the PDF method somewhat underestimates $\tau$, in agreement with [40,41]. Indeed, $\tau$ (MLE) lies between 2.4 and 2.5 for the intermediate threshold heights. This difference is, however, much less significant than in the examples [40,41]. Overall, the results provided by MLE justify the qualitative conclusions following from the PDF evaluation. The quality of fitting by the MLE method was verified by goodness-of-fit tests which generate the socalled $p$-value. As a thumb rule, a hypothesis is accepted if $p \geq 0.1$ [40] (a softer condition of $p \geq$ 0.05 is sometimes used [41]). The data presented in Table I corroborate the power-law hypothesis for the statistics of $\mathrm{AE}$ durations.

The main effect of the increase in $V_{0}$ is that the truncation of the low-amplitude signal component leads to separation of some events linked by this component and, therefore, splitting of a part of long events. As a matter of example, the maximum duration decreases from 200 to about 35 ms when $V_{0}$ is increased in the conditions of Fig. 3(a) (the minimum $\delta$ is determined by the HDT and is inferior to $0.1 \mathrm{~ms}$ for all $V_{0}$ ). It is noteworthy that the dependences of Fig. 3 show some trend to a higher probability of large durations. Such a tendency may be due to aftershocks or, perhaps, overlapping of subsequent events because of the finite strain rate, as discussed below ${ }^{1}$. The analysis

\footnotetext{
${ }^{1}$ Sound reflections correspond to very short time scales about $1 \mu$ s for an Al sample of this size [43] and must not influence the event individualization.
} 
of the aftershocks (see., e.g., [21]) goes beyond the subject of the present paper. Their possible effect on the power-law exponent can be clarified by Fig. 3(b) where the same calculations were repeated for the high HDT aiming at including all aftershocks in the triggering event. The number of detected events is lower in this case but remains significant, evolving from $1.1 \times 10^{5}$ to $4 \times 10^{3}$ when $V_{0}$ is increased. It can be seen that the power-law dependences are deteriorated at large scales. Nevertheless, approximately the same power law is detected quite reliably thus confirming that the durations of the acoustic events obey a power-law scaling. The best fit is found for an intermediate threshold level of $1.52 \mathrm{mV}: \tau \approx 2.11 \pm 0.03$ (PDF) and $\tau \approx 2.33 \pm 0.15$ (MLE) with $p=0.85$. For clarity, unrounded errors are presented as obtained from calculations.

The second main result concerns the statistics of the waiting times between AE events. Figure 4 represents probability density functions $P\left(t_{w} /<t_{w}>\right)$ for three values of strain rate and the same time settings as in Fig. 3. It should be noted that in all cases except for Fig. 4(f) corresponding to the highest $\dot{\varepsilon}_{a}$ and HDT, $N$ is gradually reduced by a factor between one and two orders of magnitude when $V_{0}$ is increased from 1.22 to $3.05 \mathrm{mV}$. Like in the above example, this depletion is governed by the removal of low-amplitude avalanches. However, as the merging of avalanches is the strongest at the highest $\dot{\varepsilon}_{a}$, selection of a high HDT in Fig. 4(f) results in a very strong merging effect and an abrupt reduction in $N$. In this case, $N$ comprises about 1400 events at $3.05 \mathrm{mV}$, grows to 6200 for $1.52 \mathrm{mV}$, and falls down to 1000 at $1.22 \mathrm{mV}$.

Very good exponential behavior is found for the lowest $V_{0}$ in the case of the low and intermediate strain rate, as can be seen in Figs. 4(a)-(d). For the set of small HDT/HLT, quite good agreement is obtained even at the highest strain rate, as shown in Fig. 4(e), although the statistical sample becomes too depleted to assure reliable analysis for the second set of time parameters in Fig. 4(f). $\chi$ hi-squared tests were performed for various choices of bin size in both cases. The tests confirmed the exponential fit with $95 \%$ confidence level for all strain rates in the case of small HDT and HLT, provided that the bin size was taken below 0.2 (the value of 0.1 was used in the presented calculations). In the case of overstated time parameters, the exponential hypothesis was also confirmed for some datasets even if it could not be verified in general. The totality of data thus allows for concluding that the waiting times obey Poisson-like statistics.

At the same time, the increase in $V_{0}$, indeed, progressively distorts the exponential dependences and results in an apparent trend to power-law behavior. Such tendencies can be recognized in all panels of Fig. 4. However, the degree of approach to a power law depends on the strain rate. The dashed line with a slope of 2 serving as a guide for the eye in Figs. 4(a) and 4(b) helps one see that neither curve obtained at the low strain rate fits a power law in an interval wider than half an order of magnitude of $t_{w}$. The fact that no reliable fit can be found is consistent with the conjecture of weak overlapping of dislocation avalanches at low strain rates, so that thresholding does not strongly contribute to separation of the events. Nevertheless, the virtual power-law scaling is satisfactory for $V_{0}=2.14 \mathrm{mV}$ at higher strain rates. Indeed, the corresponding interval exceeds one decade in Fig. 4(c) and almost reaches two orders of magnitude in Fig. 4(e). The slopes of the fitting lines, respectively, $-1.46 \pm 0.06$ and $-1.87 \pm 0.06$, correspond well to the range of scaling exponents characterizing various experimental and modeled systems (see references in Sec. I). The further increase in $V_{0}$ results in a degradation of the dependence in the small-scale range. However, the curve obtained for $V_{0}=3.05 \mathrm{mV}$ at $\dot{\varepsilon}_{a}=6 \times 10^{-3} \mathrm{~s}^{-1}$ still demonstrates a reasonably good fit over one order of magnitude of $t_{w}$. Although less convincing, these trends are also confirmed by the results obtained using the overstated time parameters, as shown in the right-hand panels of Fig. 4. Moreover, due to additional separation of AE events, the increase in $V_{0}$ results in an enrichment of the statistical sample and is associated with improved power-law dependences in Fig. 4(f). Finally, these data also corroborate the above suggestion that the effect of thresholding effect is caused by the splitting of individual events. Indeed, the scaling range found for the quiescent time distributions at high thresholds approaches the scaling range of the duration distributions at low thresholds. In the above example, the maximum $t_{w}$ increases from $40 \mathrm{~ms}$ to about $1 \mathrm{~s}$ when $V_{0}$ is increased. 
The observed trend prevents the detection of Poisson-like behavior of waiting times when the threshold is not low enough. Such a false trend may be a common reason leading to rejection of the SOC hypothesis in real experiments corrupted by noise. Furthermore, it may lead to erroneous conclusions on power-law behaviors. Verification using the MLE method with goodness-of-fit tests was performed for the datasets used to calculate the histograms of Fig. 4 . In all cases, the $p$-value was found to be equal to zero. This result allows for rejecting the power-law hypotheses for the AE data obtained in the present work. Nevertheless, it cannot be excluded that the successful invalidation of the apparent trend was aided by the narrow range of variation of waiting times in the studied phenomenon. It should be noted in this connection that the formal application of the MLE method provided estimates similar to those following from the PDF dependences.

Two remarks on the apparent PDF dependences deserve attention. The curves in Fig. 4 display imperfections in the form of bumps and depressions. As they change progressively with the $V_{0}$ variation, such distortions seem to be intrinsic to the spurious power-law PDF dependences and may be indicative of the presence of the effect of thresholding in real experiments. Similar imperfections were detected in some modeling results (cf. [24]). Another noteworthy prediction of the existing models is that a transition between two power-law exponents can take place with an increase in the threshold [44]. Such a transition may indeed be suspected for the curves obtained at $V_{0}=1.52$ and $2.14 \mathrm{mV}$ in Fig. 4. However, the present experimental data do not allow for distinguishing between the presence of two power laws and a cut-off at a large scale.

On the whole, the AE accompanying the PLC effect manifests various features of the SOC dynamics: power laws characterizing the statistics of energies [39] and durations of the hits, as well as Poisson-like behavior of quiescent times. Although the determination of the statistical distributions of the latter is less robust against the hit individualization criteria, as compared with the cases of amplitudes and durations of $\mathrm{AE}$ events, the results obtained agree well with various characteristic features of SOC dynamics. The PLC effect provides, perhaps, one of the most convincing examples of SOC in real experiments. Moreover, quantitative evidence of SOC was also found for the time series of force serrations [6,27-29]. A difference between behaviors on the scale of the deformation curve and that of AE should, however, be clarified. Power-law statistics of the force serrations were only found in the range of high strain rates, typically for $\dot{\varepsilon}_{a} \geq 10^{-3} \mathrm{~s}^{-1}$. They progressively changed to Gaussian-like distributions when $\dot{\varepsilon}_{a}$ was decreased. In Refs. [36,37], this behavior was attributed to a competition between the phenomena of SOC and synchronization in complex nonlinear systems [45]. More specifically, it is generally accepted that the collective dynamics of dislocations is mostly governed by internal stresses in the crystal lattice distorted by the dislocations themselves and other defects. In particular, this point of view is confirmed by the evolution of the AE intensity and activity accompanying the microstructure evolution during deformation, as illustrated in Fig. 2. It can be suggested that slow deformation provides enough time for the uniformization of internal stresses through non-intense dislocation processes giving rise to numerous $\mathrm{AE}$ events but is indistinguishable on deformation curves. Consequently, the building up of internal stresses brings the dislocation microstructure globally close to instability, so that the breakthrough of a dislocation group through obstacles can trigger a catastrophic deformation process involving hundreds of thousands of dislocations and resulting in a macroscopic drop in the applied force. Its size is governed by macroscopic factors such as the size and elastic properties of the system. The repetitive sequence of slow loadings followed by abrupt discharges gives rise to a relatively small number of stress serrations distributed according to Gaussian statistics. As shown in [36,37], the stress drops develop as chain processes leading to bursts in $\delta$ because of the overlapping of successive AE hits, without visible increase in the hit amplitudes. However, as the reloading time is many orders of magnitude longer than the duration of the catastrophic instability process, the number of such long AE events is negligible in comparison with their total number detected during plastic deformation. As a result, such a macroscopic instability does not bias the power-law statistics of durations of acoustic events, as shown in Fig. 3, except for contributing to the upward trend of the very right data points. 


\section{CONCLUSIONS}

In summary, a thorough analysis of the statistics of durations and waiting times was realized for the acoustic emission accompanying the unstable plastic flow. Together with the previous analysis of energy distributions [36,37], the data obtained indicate that the collective dislocation dynamics conforms to the self-organized criticality phenomenon in the scale range relevant to acoustic emission under conditions of the PLC effect. This result suggests the plastic deformation as a real system with SOC dynamics. Furthermore, the paper provides experimental evidence that thresholding leads to a transition from exponential to apparent power-law behavior of the statistics of waiting times of acoustic events. This thresholding effect does not considerably affect the experimental determination of the power-law scaling of their durations (or energies [39]). At the same time, it interferes with the determination of Poisson behavior of the waiting times. The fact that a similar transition was predicted in the literature for model systems characterized by Poisson statistics of quiescent intervals between events bears evidence to a general character of this effect that can prevent the detection of SOC-like behavior in signals corrupted by noise.

\section{ACKNOWLEDGEMENTS}

This work was supported by the Lorraine Region (France) and the French State through the program "Investment in the future" operated by the National Research Agency (ANR) and referenced by ANR-11-LABX-0008-01 (LabEx DAMAS). I.Sh. acknowledges support from the Russian Foundation for Basic Research (RFBR) through Grant No. 16-32-00264, and is grateful for the hospitality of Ecole Nationale d'Ingénieurs de Metz (ENIM) for a stay at LEM3. V.G. acknowledges the hospitality of the Université de Lorraine for a stay at LEM3.

\section{References}

[1] S. Zapperi, P. Cizeau, G. Durin, and H. E. Stanley, Phys. Rev. B 58, 6353 (1998).

[2] E.V. Matizen, S.M. Ishikaev, and V. A. Oboznov, J. Exp. Theor. Phys. 99, 1065 (2004).

[3] B. Gutenberg and C. F. Richter, Ann. Geophys. 9, 1 (1956).

[4] J.M. Carlson, J. S. Langer, and B. E. Shaw, Rev. Mod. Phys. 66, 657 (1994).

[5] M. Stojanova, S. Santucci, L. Vanel, and O. Ramos, Phys. Rev. Lett. 112, 115502 (2014).

[6] M.A. Lebyodkin, Y. Brechet, Y. Estrin, and L.P. Kubin, Phys. Rev. Lett. 74, 4758 (1995).

[7] T. Mäkinen, A. Miksic, M. Ovaska, and M.J. Alava, Phys. Rev. Lett. 115, 055501 (2015).

[8] V. Navas-Portella, Á. Corral, and E. Vives, Phys. Rev. E. 94, 033005 (2016).

[9] D.L. Turcotte, Reports on Progress in Physics 62, 1377 (1999).

[10] S. N. Dorogovtsev and A. V. Goltsev, Rev. Mod. Phys. 80, 1275 (2008).

[11] H. Kawamura, Rev. Mod. Phys. 84, 839 (2012).

[12] J.T. Uhl, Sh. Pathak, D. Schorlemmer, et al., Sci. Rep. 5, 16493 (2015).

[13] P. Bak, C. Tang, and K. Wiesenfeld, Phys. Rev. Lett. 59, 381 (1987).

[14] G. Boffetta, V. Carbone, P. Giuliani, P. Veltri, and A. Vulpiani, Phys. Rev. Lett. 83, 4662 (1999).

[15] E. Spada, V. Carbone, R. Cavazzana, L. Fattorini, G. Regnoli, N. Vianello, V. Antoni, E. Martines, G. Serianni, M. Spolaore, and L. Tramontin, Phys. Rev. Lett. 86, 3032 (2001).

[16] L.I. Salminen, A.I. Tolvanen, and M.J. Alava, Phys. Rev. Lett. 89, 185503 (2002).

[17] A. Deluca and Á. Corral, Nonlin. Proc. Geophys. 21, 555 (2014)

[18] L. Laurson and M.J. Alava, Eur. Phys. J. B 42, 407 (2004).

[19] A. Corral, Phys. Rev. Lett. 92, 108501 (2004).

[20] R. Sanchez, D.E. Newman, and B.A. Carreras, Phys. Rev. Lett. 88, 068302 (2002).

[21] J. Baró, Á. Corral, X. Illa, A. Planes, E.K.H. Salje, W. Schranz, D.E. Soto-Parra, and E. Vives, Phys. Rev. Lett. 110, 088702 (2013).

[22] K. Christensen and Z. Olami, J. Geophys. Res. 97, 8729 (1992). 
[23] M. Paczuski, S. Boettcher, and M. Baiesi, Phys. Rev. Lett. 95, 181102 (2005).

[24] L. Laurson, X. Illa, and M. J. Alava, J. Stat. Mech. - Theory Exp., P01019 (2009).

[25] E. Salje and K. A. Dahmen, Annu. Rev. Cond. Matter Phys. 5, 233 (2014).

[26] S. Janićević, L. Laurson, K.J. Måløy, S. Santucci, and M.J. Alava, Phys. Rev. Lett. 117, 230601 (2016).

[27] M.A. Lebyodkin,L.R. Dunin-Barkowskii,Y. Brechet, Y.Estrin and L.P. Kubin, Acta Mater., 48, 2529-2541 (2000).

[28] R.N. Mudrock, M.A. Lebyodkin, P. Kurath, A.J. Beaudoin, and T.A. Lebedkina, Scripta Mater. 65, 1093 ((2011).

[29] M.A. Lebyodkin, L.R. Dunin-Barkovskii, and T.A. Lebedkina, JETP Lett., 76, 612 (2002).

[30] V. S. Bobrov and M. A. Lebedkin, Fiz. Tverd. Tela 31, 120 (1989) [Sov. Phys. Solid State 31, 982 (1989)].

[31] V. S. Bobrov, S. I. Zaitsev, and M. A. Lebedkin, Fiz. Tverd. Tela 32, 3060 (1990) [Sov. Phys. Solid State 32, 1176 (1990)].

[32] J. Weiss and J. R. Grasso, J. Phys. Chem. B 101, 6113 (1997).

[33] J. Weiss, T. Richeton, F. Louchet, F. Chmelık, P. Dobron, D. Entemeyer, M. Lebyodkin, T. Lebedkina, C. Fressengeas, and R. J.McDonald, Phys. Rev. B 76, 224110 (2007).

[34] J. Weiss, W.B. Rhouma, T. Richeton, S. Dechanel, F. Louchet, and L. Truskinovsky, Phys. Rev. Lett. 114, 105504 (2015).

[35] M. Zaiser, Adv. Phys. 55, 185 (2006).

[36] M. A. Lebyodkin, N. P. Kobelev, Y. Bougherira, D. Entemeyer, C. Fressengeas, V. S.

Gornakov, T. A. Lebedkina, and I. V. Shashkov, Acta Mater. 60, 844 (2012); 60, 3729 (2012).

[37] I. V. Shashkov, M. A. Lebyodkin, and T. A. Lebedkina, Acta Mater., 60, 6842, (2012).

[38] A. Portevin and F. Le Chatelier, Comptes Rendus Acad. Sci. Paris 176, 507 (1923).

[39] M. A. Lebyodkin, I. V. Shashkov, T. A. Lebedkina, K. Mathis, P. Dobron, and F. Chmelik, Phys. Rev. E 88, 042402 (2013).

[40] A. Clauset, C. Shalizi, and M. Newman, SIAM Review, 51, 661 (2009).

[41] A. Deluca and Á. Corral, Acta Geophys. 61, 1351 (2013).

[42] S. Papanikolaou, F. Bohn, R.L. Sommer, G. Durin, S. Zapperi, and J.P. Sethna, Nature Phys.

Lett. 7, 316 (2011).

[43] M.A. Lebyodkin, T.A. Lebedkina, F. Chmelík, T.T. Lamark, Y. Estrin, C. Fressengeas, and J. Weiss, Phys. Rev. B 79, 174114 (2009).

[44] F. Font-Clos, G. Pruessner, N.R Moloney, and A. Deluca, New J. Phys. 17, 043066 (2015).

[45] C.J. Pérez, Á. Corral, A. Díaz-Guilera, K. Christensen, and A. Arenas, Int. J. Mod. Phys. B, 10, $1111(1996)$. 
Table I. Power-law exponent $\tau$ determined by least square approximation of the slope of PDF dependences in Fig. 3(a) and by the MLE method [40] applied to the same data. Numbers in parentheses indicate standard errors determined by the corresponding method.

\begin{tabular}{|c|c|c|c|}
\hline Threshold $\mathrm{V}_{0}(\mathrm{mV})$ & $\tau(\mathrm{PDF})$ & $\tau$ (MLE) & p-value \\
\hline 1.22 & $2.14(0.02)$ & $2.14(0.03)$ & 0.14 \\
\hline 1.52 & $2.22(0.03)$ & $2.40(0.13)$ & 0.53 \\
\hline 2.14 & $2.32(0.04)$ & $2.51(0.13)$ & 0.61 \\
\hline 3.06 & $1.96(0.04)$ & $2.06(0.03)$ & 0.29 \\
\hline
\end{tabular}

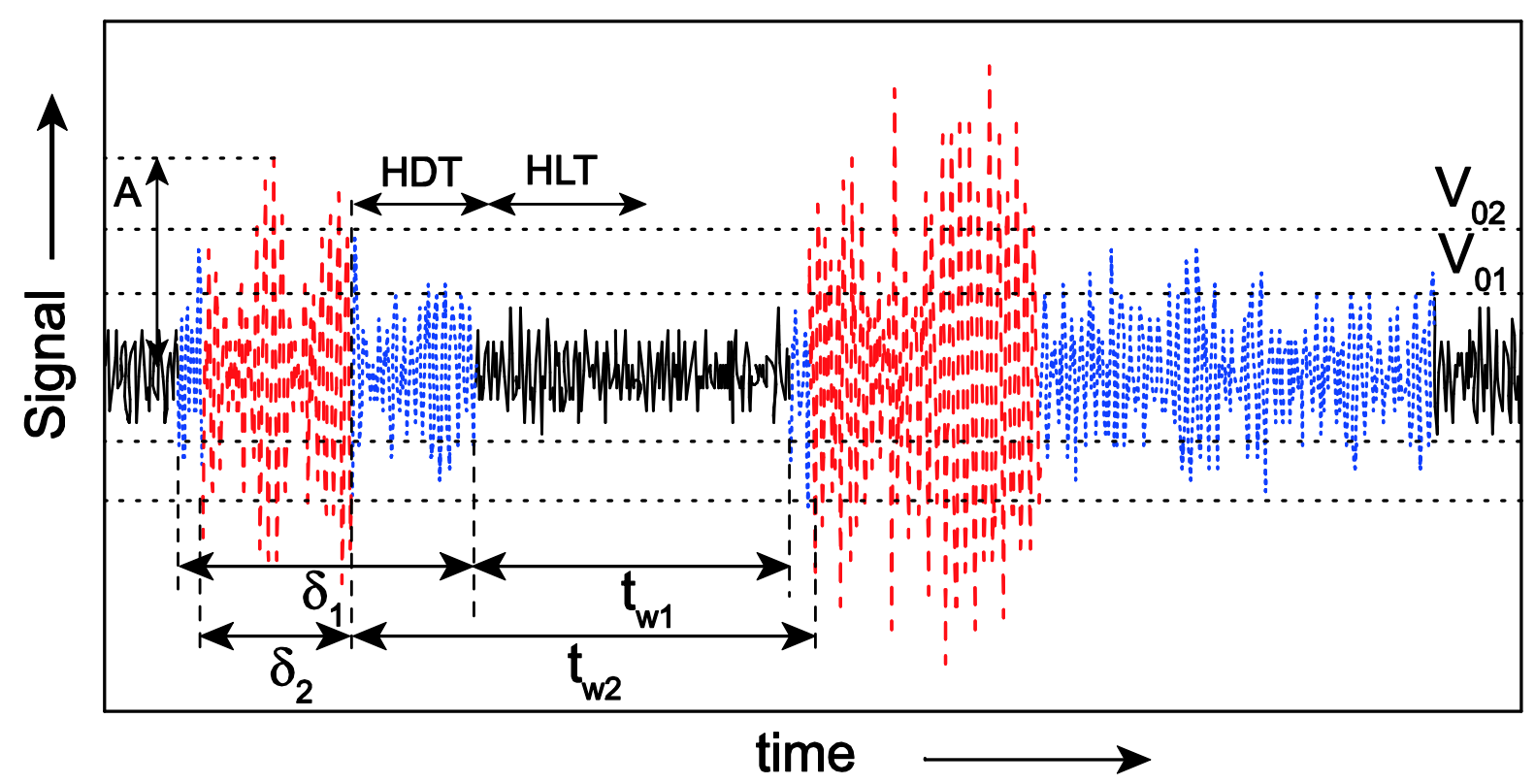

Figure 1. Scheme illustrating the effect of the threshold level $V_{0}$ on the detection of AE events. Solid line shows an example of a recorded AE signal. Dotted (blue) and dashed (red) lines display the hits found for two $V_{0}$ values. For each choice of the threshold, denoted by the order number in the subscripts, double arrows indicate the duration $\delta$ of the first of two events and the waiting time $t_{w}$ until the next one. 


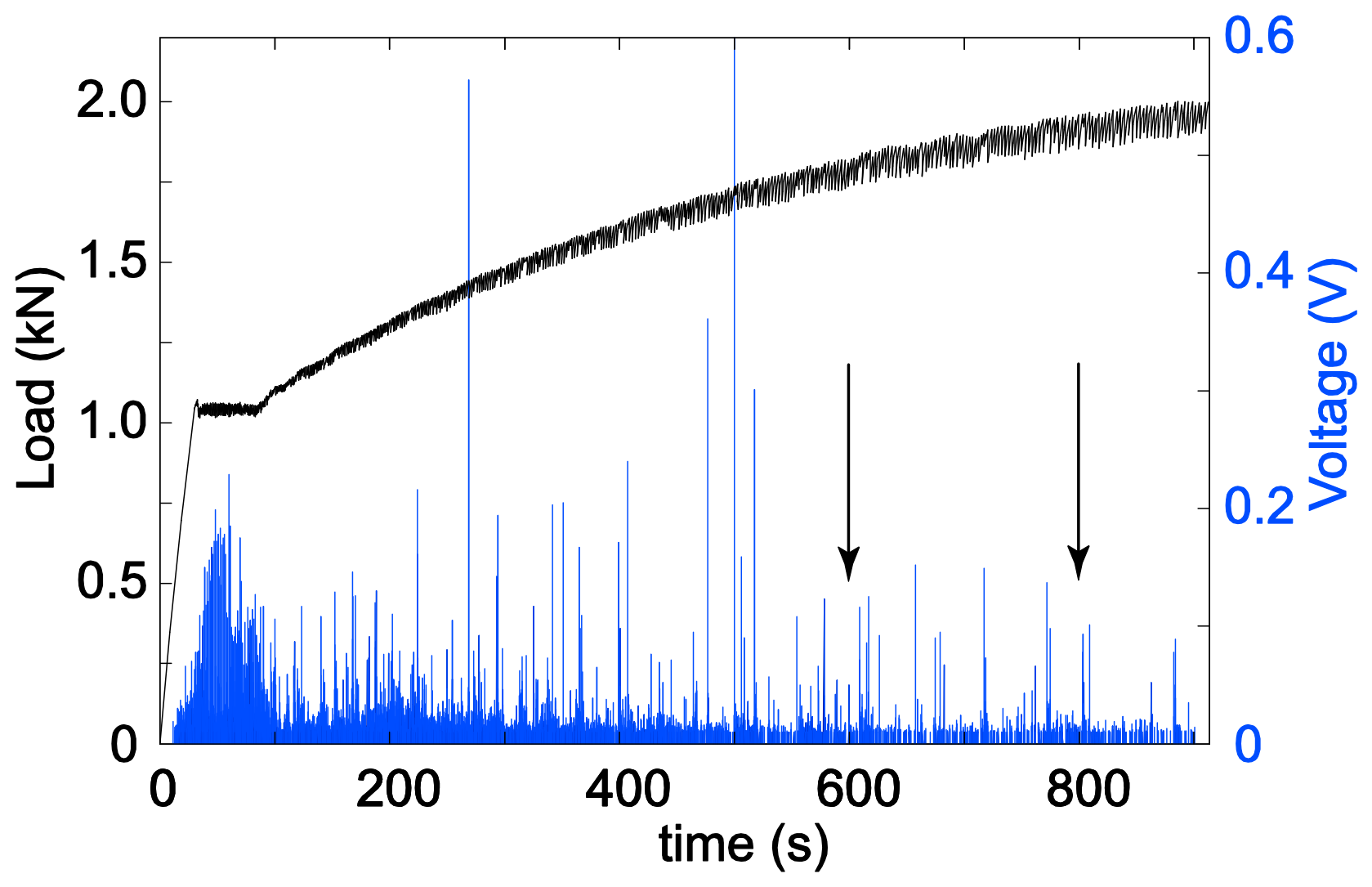

Figure 2. Example of a portion of a tensile curve recorded at $\dot{\varepsilon}_{a}=2 \times 10^{-4} \mathrm{~s}^{-1}$ and the accompanying AE signal (only the positive half-cycles are shown). Arrows indicate the time interval corresponding to the statistical analysis presented in Figs. 3 and 4(c-d).
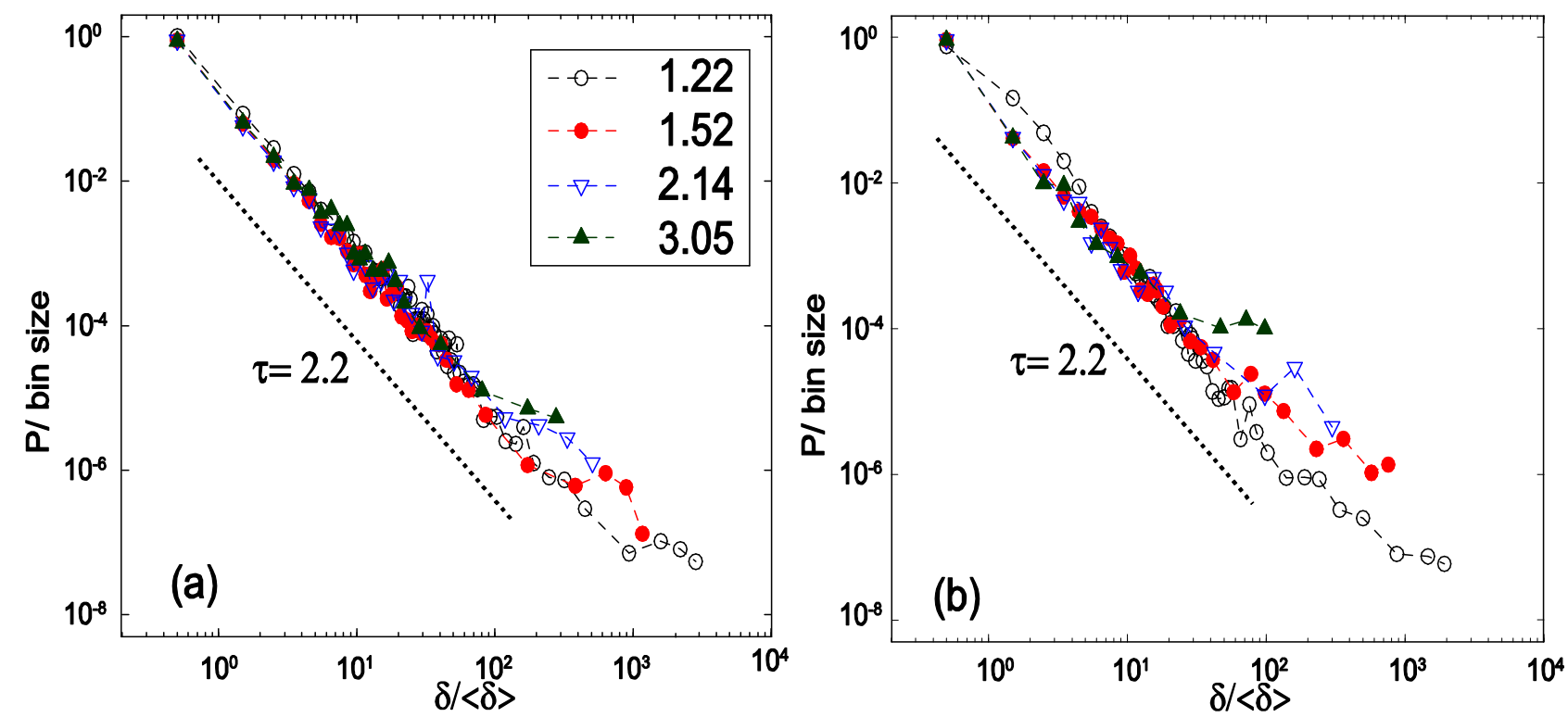

Figure 3. Probability density functions $P(\delta /<\delta>)$ normalized to the bin size for durations of AE events. (a) HDT $=30 \mu \mathrm{s}$, HLT $=100 \mu \mathrm{s}$; (b) HDT $=300 \mu \mathrm{s}$, HLT $=300 \mu \mathrm{s}$. Numbers in the legend represent the threshold $V_{0}$ in $\mathrm{mV}$. The dotted lines are provided as guides for eye and correspond to the power-law exponent $\tau=2.2$. 

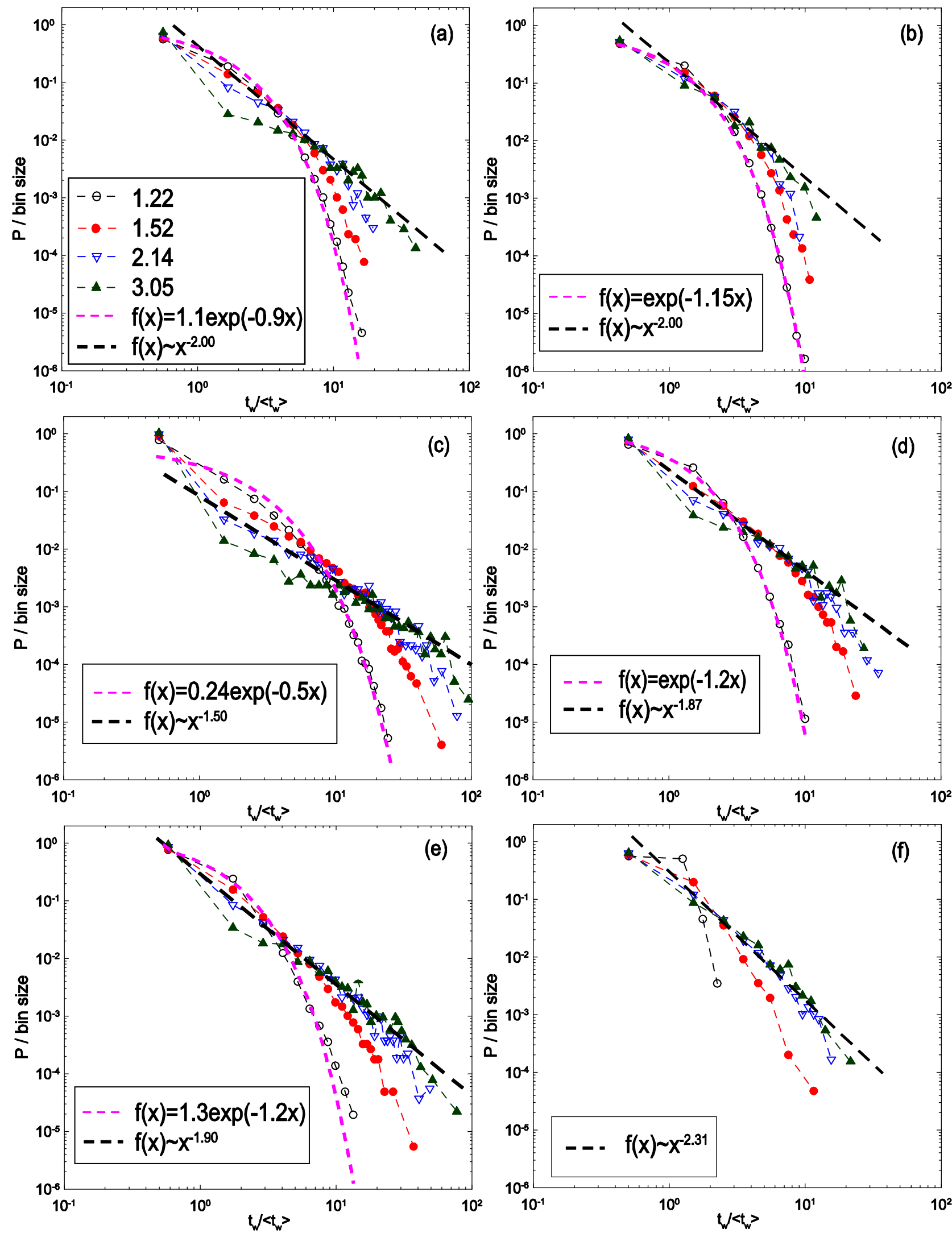

Figure 4. Effect of $V_{0}$ on the probability density functions $P\left(t_{\mathrm{w}} /<t_{\mathrm{w}}>\right)$ for inter-event waiting times. (a,b) $\dot{\varepsilon}_{a}=2 \times 10^{-5} \mathrm{~s}^{-1}$; (c, d) $\dot{\varepsilon}_{a}=2 \times 10^{-4} \mathrm{~s}^{-1}$; (e, f) $\dot{\varepsilon}_{a}=6 \times 10^{-3} \mathrm{~s}^{-1}$. Left column: HDT $=30 \mu \mathrm{s}$, HLT $=100 \mu \mathrm{s}$; right column: HDT $=300 \mu \mathrm{s}, \mathrm{HLT}=300 \mu \mathrm{s}$. Numbers in the legend of the top left plot represent the threshold values in $\mathrm{mV}$, common for all plots. The slopes of the dashed straight lines are indicated in the legends. 